\title{
Humanización
}

\section{El principialismo bioético en la humanización de la asistencia al parto}

\section{Bioethical Principlism in the Humanization of Childbirth Assistance}

Autor

\author{
Ainoa Biurrun Garrido \\ Profesora Titular en Campus Docent Sant Joan \\ de Déu. Matrona en Parc Salut Mar (Hospital del \\ Mar) \\ E-mail: ainoabiurrun@gmail.com \\ https://orcid.org/0000-0003-1955-1526
}

Carme Perelló Íñiguez

Filósofa por la Universitat de Barcelona

E-mail: carme.perello@hotmail.com

\section{Bárbara Vidal Tegedor}

Médico del Servicio de Medicina Intensiva del Hospital General Universitario de Castellón

E-mail: bategedor@gmail.com 
Resumen Los cuatro principios bioéticos descritos por Beauchamp y Childress son habitualmente utilizados para resolver problemas éticos en ciencias de la salud. En este artículo se propone su aplicación en el ámbito de la atención humanizada en la asistencia al parto. Concretamente, se plantea un análisis desde una perspectiva crítica y contextualizada del principialismo, teniendo en cuenta las limitaciones de la teoría original.

Abstract

The four principles of bioethics proposed by Beauchamp and Childress are commonly used to solve ethical issues in health sciences. This article suggests its application in the field of humanized attention to childbirth. Specifically, an analysis from a critical and contextualized perspective of principialism is proposed, considering the limitations of the original theory.

Key words Principios bioéticos; atención al nacimiento; humanización; bioética.

Bioethical principles; childbirth; humanization; bioethics.

Recibido: 31/05/2018. Aceptado: 19/09/2018 


\section{Introducción}

El principialismo es la teoría bioética más difundida y con mayor aceptación tanto en la sociedad como en el entorno de las ciencias de la salud (Seoane, 2011). Generalmente, cuando se pretende resolver problemas o dilemas éticos en ciencias de la salud se parte de sus cuatro principios, conocidos como principios bioéticos propuestos por Beauchamp y Childress en su obra de 1978 Principles of Biomedical Ethics. Los cuatro principios principialistas, que posteriormente darán paso a otros principios en otras corrientes bioéticas son: el principio de autonomía, el de no maleficencia, el de beneficencia y el de justicia. Todos ellos tienen la misma importancia por lo que se puede afirmar que no existe jerarquía y son considerados principios prima facie.

Estos principios éticos deben regir el quehacer de los profesionales de la salud y de los investigadores en general. Son principios universales e históricos que deben aplicarse en todo momento y que son de obligado cumplimiento para los profesionales sanitarios. Por tanto, y del mismo modo que estos

Todos ellos tienen la misma importancia por lo que se puede afirmar que no existe jerarquía y son considerados principios prima facie principios son básicos en todos los ámbitos de la salud, también lo son en relación a todo lo que envuelve el proceso de asistencia al nacimiento y al inicio de vida.

En las últimas décadas, uno de los términos usado con mayor profusión, en lo que a tipo de modelo de atención al nacimiento se refiere, es el de "humanización de la asistencia" que nació como respuesta al fuerte intervencionismo médico que se estaba produciendo sobre un proceso fisiológico como es el parto y que habría cuestionado en algunos aspectos los cuatro principios mencionados (Biurrun-Garrido \& Goberna-Tricas, 2013).

Hasta finales del siglo XIX parir en un hospital era considerado signo de pobreza dado que se asociaba a mayor peligro para la vida de la mujer por la alta mortalidad materna debida, en gran medida, a las fiebres puerperales (Goberna-Tricas, 2009). No obstante, esta situación cambia a partir del siglo XX como consecuencia de la revolución biomédica de occidente provocada por las mejoras sociales, económicas y por los progresos en descubrimientos, conocimientos y tecnologías sanitarias que repercutieron directamente sobre la disminución de la morbimortalidad materna e infantil (Biurrun-Garrido \& Goberna-Tricas, 2017).

A partir de entonces, se producen importantes avances en el ámbito de la ginecología y la obstetricia que permiten solucionar o minimizar problemas de salud. Sin embargo, estos avances suelen anteponer la tecnificación y el conocimiento científico a las necesidades de las usuarias, justificándose esta conducta en la mejora de los resultados obstétricos (Heidegger, 2007). Asimismo, el aumento de los protocolos asistenciales durante la atención al nacimiento ha comportado, en numerosas circunstancias, la realización de praxis no siempre justificadas desde una perspectiva de "práctica basada en la evidencia científica" obviando en numerosas ocasiones las particularidades que se producen en cada proceso de atención al nacimiento. También hay que mencionar que el avance de la ciencia y la tecnología provoca un aumento de las especializaciones 
profesionales existentes, creando incluso subespecializaciones, influyendo a su vez en la estructuración de los servicios sanitarios, contratación y capacitación de profesionales que acompañan el proceso.

Debe tenerse en consideración que el parto es un proceso fisiológico y no una patología o enfermedad en la cual los sanitarios deban intervenir para restablecer la salud de la usuaria; estamos hablando de la atención de un proceso orgánico en el que una persona, una mujer, "da a luz" a otro ser humano, a otra persona. En el caso de la atención al nacimiento es importante puntualizar que los cuidados deben

En el caso de la atención al

nacimiento es importante

puntualizar que los cuidados

deben ser dirigidos a

dos seres que necesitan

atenciones individualizadas y

personalizadas ser dirigidos a dos seres que necesitan atenciones individualizadas y personalizadas, dado que sus características personales las hacen únicas al igual que lo será su proceso de parto y la vivencia del mismo. Esto hace necesario que los cuidados o atenciones además de estar basados en la evidencia, respeten la dignidad de la persona (Torralba Roselló, 2005).

Pese a tratarse de un proceso fisiológico, el trabajo de parto se ha relacionado con el concepto de riesgo ya que potencialmente lo presenta, tanto para la madre como para el feto o para el recién nacido. Muestra de ello se encuentra en el informe de la Organización Mundial de la Salud (OMS) (World Health Organization, UNICEF, UNFPA, World Bank Group, \& United Nations, 2015), que estima que en el año 2015 murieron en el mundo 303.000 mujeres durante el embarazo y el parto. Asimismo, la OMS puntualiza que el $99 \%$ de dichas muertes se producen en países en vías de desarrollo (con 302.000 fallecimientos) donde no se suele disponer de profesionales conocedores de prácticas obstétricas ni, en numerosas ocasiones, de una mínima infraestructura asistencial. Además, actualmente en el mundo occidental existe una tendencia a posponer la edad de primiparidad, incrementándose esta en la mayoría de países. Según la Organización para la Cooperación y el Desarrollo Económicos (OCDE) en 2015 la edad media de primer parto se sitúa en una media de 30,1 años (OECD, 2018). Esto es debido en gran medida a los cambios socioculturales que ha experimentado el rol de la mujer en la sociedad, circunstancia que aumenta el riesgo potencial de complicaciones obstétricas, morbimortalidad y medicalización del parto, e incrementa la necesidad de individualizar y humanizar los cuidados (Cavazos-rehg et al., 2015).

En este artículo se plantea cómo es posible proporcionar un cuidado individual y humanizado, cumpliendo las expectativas de las usuarias desde un análisis basado en el principialismo bioético.

\section{El principialismo en la humanización del parto}

Tal y como ya se ha anunciado anteriormente, el principialismo propone 4 principios bioéticos: el principio de autonomía, el de beneficencia, el de no maleficencia y el de justicia. A continuación, se analizará la aplicación de los principios propuestos por Beauchamp y Childress en la atención al nacimiento. 


\subsection{El principio de autonomía}

El respeto a la autonomía se sustenta en el reconocimiento del derecho legítimo del individuo y en la capacidad de este para tomar decisiones libremente. El derecho de las personas a tomar decisiones relativas a su salud y sobre su vida, aparece con la asunción de la ideología liberal en la sociedad y, como no, en la práctica clínica. Somos autónomos no solo porque somos humanos, sino para ser más humanos (Goberna-Tricas \& Boladeras Cucurella, 2016); en eso nos refuerza el principio de

El principio de autonomía obliga al profesional sanitario a respetar los valores y creencias del usuario, considerando las perspectivas que estos tienen sobre sus intereses y utilizando únicamente estrategias clínicas autorizadas por parte del paciente, como resultado de un proceso de consentimiento informado autonomía. No obstante, si nos centramos en la autonomía en la toma de decisiones y en las opciones de la mujer durante el parto, la dignidad y la autonomía de la gestante se hacen patentes a través de su derecho a recibir atenciones y cuidados, y en el derecho a adoptar decisiones autónomas ${ }^{1}$.

El respeto al principio de autonomía se lleva a cabo, fundamentalmente, a través del ejercicio del consentimiento libre (sin restricciones o presiones indebidas) e informado (después de proveerse la información objetiva, veraz y apropiada a la acción propuesta). Además, la autonomía contempla que la persona pueda decidir hasta donde ser informada y que pueda cambiar su opinión en cuanto al consentimiento en cualquier momento. Es preciso recordar que el derecho a retirar su consentimiento se encuentra consagrado en el artículo 5 del Convenio de Oviedo, conocido también como Convenio de Asturias o Convenio sobre los Derechos Humanos y Biomedicina de 1997. El respeto al principio de autonomía constituye el núcleo central en el respeto a la toma de decisiones. Beauchamp y Childress definen autonomía como "la capacidad de autogobierno que posee la propia persona y que incluye la comprensión, el razonamiento, la reflexión y la elección independiente". Así pues, el principio de autonomía obliga al profesional sanitario a respetar los valores y creencias del usuario, considerando las perspectivas que estos tienen sobre sus intereses y utilizando únicamente estrategias clínicas autorizadas por parte del paciente, como resultado de un proceso de consentimiento informado.

El consentimiento informado, que se precisa para la ejecución de este principio de autonomía, permite garantizarla mediante la autodeterminación y el respeto a los individuos involucrados en el proceso de investigación científica o que reciben atención médica. No obstante, para que se produzca realmente un consentimiento informado se debe dar una información comprensible, objetiva y veraz al usuario de los servicios sanitarios, que en este caso sería la gestante, parturienta o puérpera, además de existir una

1 Ciertamente, en el tema que nos ocupa podría surgir la reflexión acerca de la autonomía del feto. Por lo que debería plantearse la cuestión de si se considera al feto un sujeto de derecho con autonomía o no. Ello implicaría que el principio de autonomía tendría doble consideración; por una parte, debería considerarse la autonomía de la gestante y, por otro lado, la autonomía del feto. Al hablar de autonomía del feto se podría debatir si este tiene posibilidad de consentimiento por representación y, de ser así quién debería ejercerla en ese caso. 
comprensión de dicha información por parte de la usuaria y una aceptación o voluntariedad en aceptar el procedimiento o cuidados que se le van a realizar.

En España, la Estrategia de Atención al Parto Normal hace referencia a la importancia de asegurar y garantizar el acceso y comprensión de la información de las mujeres sobre la fisiología del parto y sobre las prácticas clínicas de atención al nacimiento. Además, en dicha guía se comenta la importancia de que la información sea completa y que en ella se consideren las razones, los beneficios, los riesgos y los resultados oportunos a las necesidades de las mujeres en relación al lenguaje, formato y momento en el que dar la información. Asimismo, la estrategia remarca la importancia de dar información basada en la evidencia, con la finalidad de permitir a las mujeres la toma de decisiones informadas de acuerdo a sus necesidades y expectativas. La guía también menciona la necesidad de respetar las decisiones que tome la mujer acerca de las actuaciones de su parto, mediante instrumentos como el plan de parto o plan de nacimiento; documento elaborado durante el embarazo junto al asesoramiento de profesionales sanitarios, para garantizar decisiones autónomas en un

El ser humano no está solo en el mundo, el ser humano tiene una vertiente relacional que afecta directamente sobre el concepto "autonomía" marco de seguridad y calidad asistencial, junto a la garantía del reconocimiento del derecho de la madre y el padre o tutor legal sobre cualquier decisión en relación a la criatura recién nacida.

Además, en el principio de autonomía aplicado al proceso de parto, se ha de tener en cuenta que vivimos en una sociedad plural. Esto es de vital importancia a la hora de realizar las comunicaciones a las mujeres que pasarán por un proceso de parto, ya que una correcta escucha activa y una comprensión por ambas partes será la clave para decidir en libertad y para llegar a poder respetar la dignidad ontológica de la usuaria.

También, en este caso, nos encontramos con una cuestión de género que es fundamental. Es en ese sentido que el feminismo redefine el concepto de autonomía como autonomía relacional (Álvarez, 2015). ¿A qué se refiere este nuevo concepto? A la inclusión de la visión del individuo no como un ser aislado del mundo y desvinculado de su entorno, sino como un ser que se interrelaciona y que es interdependiente. El ser humano no está solo en el mundo, el ser humano tiene una vertiente relacional que afecta directamente sobre el concepto de "autonomía".

La autonomía del individuo debe tener en cuenta a las personas que forman parte de la vida de este. Existen relaciones, compromisos, vínculos que deben quedar contemplados en el ejercicio de esta autonomía. En el caso que nos atañe, se debe tener en cuenta el entorno en el que se produce el nacimiento y a las personas que acompañan a la usuaria en dicho proceso.

Se ha hecho mucho énfasis en la vertiente más independiente y desvinculada de la comunidad de la autonomía; pero la persona vive en sociedad, que es la que otorga identidad y sentido a la misma, por lo que finalmente el objetivo es que la usuaria ejerza su autonomía relacional. Este aspecto, para el feminismo, en tanto que defiende los intereses de los oprimidos, el contexto social y comunal es altamente importante. No se debe olvidar que los colectivos oprimidos no son totalmente libres y autóno- 
mos en sus contextos, ya que están sujetos a las normas y los valores del colectivo opresor.

En la bioética "tradicional" se observan las normas y los valores del colectivo opresor, y es por eso por lo que es tan necesaria la revisión de los conceptos en una base feminista. Se han de releer los preceptos bioéticos "tradicionales" y ajustarlos a las necesidades de los colectivos oprimidos.

Los colectivos oprimidos no son totalmente libres y autónomos

en sus contextos, ya que están

sujetos a las normas y los

valores del colectivo opresor

También observamos que en todo lo que se refiere a las leyes establecidas en relación a la salud de la mujer (reproducción asistida, interrupción del embarazo...) el concepto autonomía queda totalmente reducido, ya que aquello que es de orden personal pasa a ser de orden político.

En un principio podría creerse que en un entorno neoliberal el principio de autonomía no debería de encontrar ningún obstáculo para su aplicación, pero no es así. El principio de autonomía se ha encontrado con desafíos importantes dentro de este entorno. ¿Cómo se puede aplicar el principio de autonomía en sistemas que mantienen prácticas de dominio? El enfoque de género, el feminismo, ha hecho visible esta incongruencia del sistema y debe continuar trabajando en este sentido. El dominio patriarcal resta fuerza de aplicación a los principios universales, ya que establece dos niveles de autonomía; uno, el androcéntrico, donde la autonomía es plena, y otro, el de los grupos oprimidos, que están sujetos a las condiciones sociales, de comunidad e incluso políticas.

\subsection{El principio de beneficencia}

El modelo de relación asistencia predominante durante la historia de la medicina ha sido el paternalismo (Lázaro \& Gracia, 2006). Desde el siglo V a. de C. el Juramento Hipocrático ha descrito la forma en la que el médico debía tratar al paciente. Cabe mencionar que, la actitud hipocrática considera al usuario como un ser incapaz de tomar decisiones autónomas y esta actitud se ha mantenido hasta la edad contemporánea. No obstante, a partir del siglo XX la relación profesional sanitario-usuario ha ido cambiando, y esta transformación también se ha reflejado en lo que a asistencia al nacimiento se refiere. Se ha pasado de un modelo paternalista, de relación vertical en el que limitaba de manera intencionada la libertad de decisión y acción, siendo en numerosas ocasiones autoritario al no tomar en cuenta el parecer del usuario, justificándose en la evitación de un daño o proporcionarle un bien, a un modelo basado en la autonomía, más horizontal y más participativo. Asimismo, también ha cambiado la relación interprofesional que se establece entre los diferentes profesionales implicados en la asistencia al nacimiento: ginecólogos, tocólogos, ecografistas, enfermeras, matronas, auxiliares de enfermería, camilleros, administrativos... El modelo clásico, caracterizado por la subordinación, sumisión y obediencia de los diferentes profesionales al médico, se ha modificado hacia un modelo de equilibrio y armonización de roles profesionales. Por ello, hoy en día, se puede afirmar que en la práctica clínica se establece un modelo 
de colaboración multiprofesional e interprofesional. Cada profesional conoce y tiene descritas sus competencias profesionales y procura relaciones de respeto y de armonía con los otros, tratando de trabajar como iguales.

Es preciso recordar que el principio de beneficencia exige que hagamos pasos para ayudar a los demás, y no únicamente que nos abstengamos de actos perjudiciales. Beauchamp y Childress hablan de dos principios de beneficencia: la beneficencia positiva y la utilidad (Siurana, 2010). Los definen así: "La beneficencia positiva requiere la aportación de beneficios (obligación de

El principio de beneficencia

exige que hagamos pasos

para ayudar a los demás

y no únicamente que nos

abstengamos de actos

perjudiciales proporcionar unos beneficios). La utilidad exige que los beneficios y los inconvenientes están equilibrados".

En lo que a asistencia al nacimiento se refiere se puede objetivar la beneficencia positiva en el deber que el profesional sanitario tiene a hacer un bien al usuario, de procurarle un beneficio, obligación que se desprende de lo que se expone en los códigos deontológicos de cualquiera de las profesiones sanitarias. Por otro lado, la beneficencia de utilidad es una extensión de la anterior, para aquellos casos en los que tratar o cuidar a una usuaria hace que entren en juego riesgos y beneficios. Es preciso recordar que muchas de las acciones que los profesionales sanitarios llevan a cabo no están libres de consecuencias negativas, por lo que ante una intervención siempre debe valorarse si existe riesgo de iatrogenia al realizarla y valorar si procede realmente la aplicación de la misma. En referencia al nacimiento, siempre se debe valorar antes de llevar a cabo cualquier técnica, incluso al hacer un tacto vaginal en una gestante a término con bolsa íntegra, si es preciso la realización de ese procedimiento, puesto que llevar a cabo cualquier técnica, aunque se realice por parte de un profesional prudente y experto, podría conllevar riesgos. En el caso presentado podría estimular, como riesgo potencial o secundario al procedimiento, la estimulación al parto.

\subsection{El principio de no maleficencia}

A pesar de que en un primer momento se pensó que si se hacía el bien a un enfermo no cabía hacerle un mal, la evolución de la práctica asistencial ha hecho ver que eso no siempre es así, por ello se ha precisado remarcar que en aquellos casos en los que no es posible hacer un bien, dado que el conocimiento sanitario tiene sus limitaciones, el profesional debe preocuparse por no hacer un mal o un daño. Este hecho se recoge ya en la consigna latina de ética hipocrática o ética médica: primum non nocere (Júdez, 2001).

Los profesionales sanitarios deben no perjudicar al paciente o usuario/a. El profesional sanitario debe tratar de hacer el bien, pero en caso de no poderlo llegar a conseguir, procurar no producir un mal. Este principio obliga a no hacer daño a los pacientes; a las gestantes o usuarias de los servicios de asistencia al nacimiento, ni a los fetos o neonatos.

Además, este principio también contempla que, si por cualquier circunstancia se llegara a provocar un mal, este sea el menor mal necesario para conseguir un bien mayor. 
Pero desgraciadamente esto no siempre ha sido así. A lo largo de la historia de la obstetricia se ha podido observar en algunas ocasiones tratos maleficentes proporcionados por los profesionales sanitarios, los cuales se han enmarcado bajo el concepto de "violencia obstétrica". Pero ¿qué significa este concepto? El término "violencia obstétrica" es relativamente moderno y hace referencia a la violencia llevada a cabo por los profesionales de la salud sobre el cuerpo y los procesos reproductivos de las mujeres (Belli, 2013). La relación

A lo largo de la historia de

la obstetricia se ha podido

observar, en algunas

ocasiones, tratos maleficentes

proporcionados por los

profesionales sanitarios, los

cuales se han enmarcado

bajo el concepto de "violencia

obstétrica" asimétrica que se establece entre los profesionales sanitarios y las mujeres llega a reducir la capacidad de estas de valerse por sí mismas, restándoles protagonismo durante el embarazo, parto y puerperio. Además, es preciso remarcar que la violencia obstétrica es entendida como una forma de violación de los derechos humanos y a pesar de ello muchas veces es ignorada (Alvarado Rigores \& Guerra, 2011). Algunos autores (Casal-Moros \& Alemany-Anchel, 2014) muestran que la violencia obstétrica se manifiesta como una violencia simbólica, puesto que se ejerce sin mediación de la fuerza física, sin que el agente dominado sea consciente de su estado de sumisión y sin que se sienta obligado a actuar. La usuaria de los servicios de maternidad, en numerosas ocasiones, ha adoptado un papel pasivo, sin cuestionar las indicaciones o las intervenciones a las que es sometida.

El poder simbólico es un poder "invisible", que no es reconocido como tal, sino como algo legítimo. La normalización de este poder, asumir que la situación tiene que ser así y que no puede ser de otra manera, es lo que se debe erradicar (Delgado Rodríguez, 2016). Por ello, actualmente, en algunos países ha aparecido legislación para procurar regular y penalizar este tipo de violencia.

\subsection{El principio de justicia}

Este es el que establece que tratemos a todas las personas con la misma consideración y respeto (los iguales deben ser tratados igualmente), y que distribuyamos de manera equitativa los recursos y las cargas en materia sanitaria para evitar discriminaciones entre los ciudadanos. En el concepto de justicia se observa que la justicia equivale a dar a cada uno lo que le corresponde. La justicia debe interpretarse como trato igualitario, equitativo y apropiado a la luz de lo que se debe a las personas o es propiedad de ellas.

Aristóteles, entendía la justicia como virtud perfecta (Garcés Giraldo \& Giraldo Zuluaga, 2014), y desarrolló una definición (definición aristotélica) del concepto de justicia mediante la cual explicó que esta toma dos formas particulares; la justicia distributiva y la conmutativa. La justicia distributiva se refiere a los principios que regulan la distribución del honor o el dinero u otros activos divisibles de la comunidad. En el caso de la sanidad y de la atención al nacimiento, que se ejerza una correcta justicia distributiva a la hora de realizar un adecuado control durante la atención al nacimiento y una apropiada atención al parto y puerperio resulta transcendental para que se puede 
disponer de un sistema sanitario que cubra las necesidades de los usuarios y garantice una atención adecuada y de calidad. La adquisición de las competencias para hacer una gestión ética de los recursos pasa por reconocer el derecho a la salud como parte de la justicia social, entender que la eficiencia en las organizaciones sanitarias es un instrumento para alcanzar la justicia distributiva y practicar una atención efectiva y de calidad (Coronado-Vázquez \& Gómez-Salgado, 2018).

\section{Conclusiones}

En este artículo se ha realizado un análisis bioético de la humanización al parto a partir de una perspectiva crítica y contextualizada del principialismo, presentando las limitaciones de la teoría original para el análisis del parto humanizado y destacando las contribuciones de algunos investigadores para una mejor interpretación del tema. Primero, referente al principio de autonomía, observamos la necesidad de respetar los valores y creencias del usuario, cuya máxima expresión es el consentimiento informado; herramienta que permite la ejecución de una autodeterminación.

El principio de beneficencia

nos recuerda la obligación

e importancia de los

profesionales de la salud en

proporcionar un beneficio

minimizando los actos

perjudiciales
En España, la Estrategia de Atención al Parto Normal, resalta la importancia de la autonomía proporcionando una serie de recomendaciones en cuanto a prácticas clínicas, comunicación y respeto a las decisiones de la mujer.

El principio de beneficencia nos recuerda la obligación e importancia de los profesionales de la salud en proporcionar un beneficio minimizando los actos perjudiciales. Un estudio profundo bajo este principio requeriría un análisis concreto de los cuidados y técnicas sanitarias. No obstante, algunas prácticas sanitarias que a priori parecen inocuas pueden comportar un riesgo iatrogénico para las gestantes. Ante esto, debemos plantearnos en cada caso la Lex Artis ad hoc individualizando los protocolos y haciendo que los profesionales reflexionen sobre la práctica clínica.

En cuanto al principio de no maleficencia en lo que a atención al nacimiento se refiere, los profesionales sanitarios tienen la obligación de, al menos, no perjudicar a la mujer, feto, recién nacido o neonato, cuando no sea posible hacer el bien. En contraposición a este principio destaca el concepto de "violencia obstétrica"; prácticas consideradas violentas durante la atención al nacimiento por los profesionales sanitarios y cuyas implicaciones han hecho necesaria su legislación en algunos países.

En referencia al principio de justicia, cabe destacar la importancia de la justicia distributiva, dado que en cualquier relación asistencial es importante tener en cuenta la equidad de los recursos profesionales y estructurales para proporcionar una atención sanitaria equitativa e igualitaria.

La implementación de los principios bioéticos en la práctica asistencial, tal y como se ha descrito durante este artículo, puede ayudar a mejorar la calidad de la atención y, sobre todo, la humanización de la asistencia. Para ello es preciso que se integren los 
principios bioéticos en el ejercicio profesional y de ese modo se aplique la bioética para la humanización del parto.

\section{Bibliografía}

Alvarado Rigores, M., \& Guerra, N. (2011). La violencia de género un problema de salud pública. Interacción y Perspectiva: Revista de Trabajo Social, 2(2), 97-106.

Álvarez, S. (2015). La autonomía personal y la autonomía relacional. Análisis Filosófico, 35(1), 13-26.

Belli, L. F. (2013). La violencia obstétrica: otra forma de violación a los derechos humanos. Revista Redbioética/UNESCO, 1(7), 25-34.

Biurrun-Garrido, A., \& Goberna-Tricas, J. (2013). La humanización del trabajo de parto: necesidad de definir el concepto. Revisión bibliográfica. Matronas Profesión, 2, 62-66.

Biurrun-Garrido, A., \& Goberna-Tricas, J. (2017). Buenas prácticas y participación informada de la mujer en la atención al parto. Viure En Salut, 111, 17-18.

Casal-Moros, N., \& Alemany-Anchel, M. J. (2014). Violencia simbólica en la atención al parto. Un acercamiento desde la perspectiva de Bourdieu. Index Enferm (Gran), 23(12), 61-64.

Cavazos-rehg, P. A., Krauss, M. J., Spitznagel, E. L., Bommarito, K., Madden, T., Olsen, M. A., ... Bierut, L. J. (2015). Maternal age and risk of labor and delivery complications Patricia. Matern Child Health J, 19(6), 1202-1211. DOI: https://doi.org/10.1007/s10995-014-1624-7

Coronado-Vázquez, V., \& Gómez-Salgado, J. (2018). Enseñanza de ética en la distribución de los recursos y la protección de la salud para una atención equitativa y segura. Educación Médica. DOI: https://doi. org/10.1016/j.edumed.2018.01.002

Delgado Rodríguez, J. (2016). Vulnerabilidad y fragilidad. La importancia de los vínculos. En M. Boladeras Cucurella \& J. Goberna-Tricas (Eds.), Bioética de la maternidad: humanización, comunicación y entorno sanitario (1. a ed., pp. 91-100). Barcelona: Publicacions de la Universitat de Barcelona.

Garcés Giraldo, L. F., \& Giraldo Zuluaga, C. (2014). Virtudes éticas en Aristóteles: razón de los deseos y sus acciones para lograrlas. Revista Virtual Universidad Católica Del Norte, (41), 70-78.

Goberna-Tricas, J. (2009). Dones i procreació: Ėtica de les pràctiques sanitàries i la relació asistencial en embaràs i naixement (Tesis doctoral). Universitat de Barcelona.

Goberna-Tricas, J., \& Boladeras Cucurella, M. (Eds.) (2016). Bioética de la maternidad: humanización, comuni-



Heidegger, M. (2007). Filosofía, ciencia y técnica. Universitaria.

Júdez, J. (2001). La deliberación moral: el método de la ética clínica. Medicina Clínica, 117(1), 18-23. DOI: https://doi.org/10.1016/S0025-7753(01)71998-7

Lázaro, J., \& Gracia, D. (2006). La relación médico-enfermo a través de la historia. Anales del Sistema Sanitario de Navarra, 29, 7-17.

OECD. (2018). SF2.3: Age of mothers at childbirth and age-specific fertility. 
Seoane, J. A. (2011). Bioética y humanización del parto. En Estudios multidisciplinares para la humanización del parto. Jornadas Universitarias Multidisciplinares para la Humanización del Parto (pp. 87-101). A Coruña: Universidade da Coruña, Servizo de Publicacións.

Siurana, J. C. (2010). Los principios de la bioética y el surgimiento de una bioética intercultural. Veritas: Revista de Filosofía y Teología, (22), 121-157.

Torralba Roselló, F. (2005). ¿Qué es la dignidad humana? Ensayo sobre Peter Singer, Hugo Tristram Engelhardt y John Harris. Herder.

World Health Organization, UNICEF, UNFPA, World Bank Group, \& United Nations. (2015). Trends in maternal mortality, 1990 to 2015 . Retrieved from

http://apps.who.int/iris/bitstream/10665/194254/1/9789241565141_eng.pdf 